\title{
From Design to Implementation: an Automated, Credible Autocoding Chain for Control Systems
}

\author{
Timothy Wang ${ }^{1}$, Romain Jobredeaux ${ }^{1}$, Heber Herencia ${ }^{2}$, Pierre-Loïc Garoche ${ }^{3}$, \\ Arnaud Dieumegard ${ }^{4}$, Éric Féron $^{1}$, and Marc Pantel ${ }^{4}$ \\ ${ }^{1}$ Georgia Institute of Technology, Atlanta, Georgia, USA \\ ${ }^{2}$ National Institute of Aerospace, Virginia, USA \\ ${ }^{3}$ ONERA - The French Aerospace Lab, Toulouse, FRANCE \\ ${ }^{4}$ ENSEEIHT, Toulouse, France
}

\begin{abstract}
This article describes a fully automated, credible autocoding chain for control systems. The framework generates code, along with guarantees of high level functional properties which can be independently verified. It relies on domain specific knowledge and fomal analysis to address a context of heightened safety requirements for critical embedded systems and ever-increasing costs of verification and validation. The platform strives to bridge the semantic gap between domain expert and code verification expert. First, a graphical dataflow language is extended with annotation symbols enabling the control engineer to express high level properties of its control law within the framework of a familiar language. An existing autocoder is enhanced to both generate the code implementing the initial design, but also to carry high level properties down to annotations at the level of the code. Finally, using customized code analysis tools, certificates are generated which guarantee the correctness of the annotations with respect to the code, and can be verified using existing static analysis tools. While only a subset of properties and controllers are handled at this point, the approach appears readily extendable to a broader array of both.
\end{abstract}

Keywords: Control Engineering, Autocoding, Lyapunov proofs, Formal Verification, Control Software

A wide range of today's real-time embedded systems, especially their most critical parts, relies on a control-command computation core. The control-command of an aircraft, a satellite, a car engine, is processed into a global loop repeated forever, or at least during the activity of the controlled device. This loop models the acquisition of new input values via sensors: either from environment mesures (wind speed, acceleration, engine RPM, ...) or from the human feedback via the brakes, the accelerator, the stick or wheel control.

The cost of failure of such systems is tremendous, and examples of such failures abound, in spite of increasingly high certification requirements. Current 
analysis tools focus mainly on simulations. One obvious shortcoming is the impossiblity to simulate all the possible scenarios the system will be subject to. More advanced tools include static analysis modules, which derive properties of the system by formally analyzing its semantics. However, in the specific case of control systems, analyzing the computational core can prove arduous for these tools, whereas the engineers who designed the controller have a variety of mathematical results which can greatly facilitate said analysis, and evince more subtle properties of the implemented controller.

This article, following previous efforts aimed at demonstrating how controlsystem domain knowledge can be leveraged for code analysis [5] [7], attempts to describe a practical implementation of a fully automated framework, which enables a control theorist to use familiar tools to generate credible code, that is, code delivered with a certificate ensuring certain properties will hold on all executions.

This article focuses on a specific class of controllers and properties in order to achieve full automation, but also explores various possible extensions.

The structure of the article is as follows: We first present a high level view of the general framework in Section 1. We then proceed to describe how control semantics can be expressed at different levels of design, in Section 2. Section 3 describes the translation process by which graphical synchronous languages familiar to the control theorist can be turned into credible code. Section 4 demonstrates how a proof of correctness can be automatically extracted from the generated code.

\section{Framework and the Running example}

The framework of credible autocoding of control software using control semantics, which led to the development of our prototype, is summarized in Figure 1. This novel framework represents a possible conduit that will allow the domain expert e.g. the control engineer to more efficiently produce code with automatically certifiable safety and high-level functional properties. Compared to the typical model-based development paradigm, the only additional requirement on the control engineer that our framework stipulates is the need for the proofs of high-level control system properties such as Lyapunov stability, vector margins, and other performance measures to be provided in the high-level specifications of the control system.

The process of generating the proofs can be automated using techniques from the robust control literature. See works such as [13], [14], [5], or [10].

The are two major branches in the framework. In the first branch, the input model along with its control semantics, is directly translated to the annotated source code. On the source code level, the annotations are further translated into proof obligations, which are then discharged by a software theorem prover. To demonstrate an automation of the first branch, we built a set of specialized prototype tools, which include an autocoder that generates the control semantics, and an annotation checker that is tailored for verifying the ellipsoid-based 


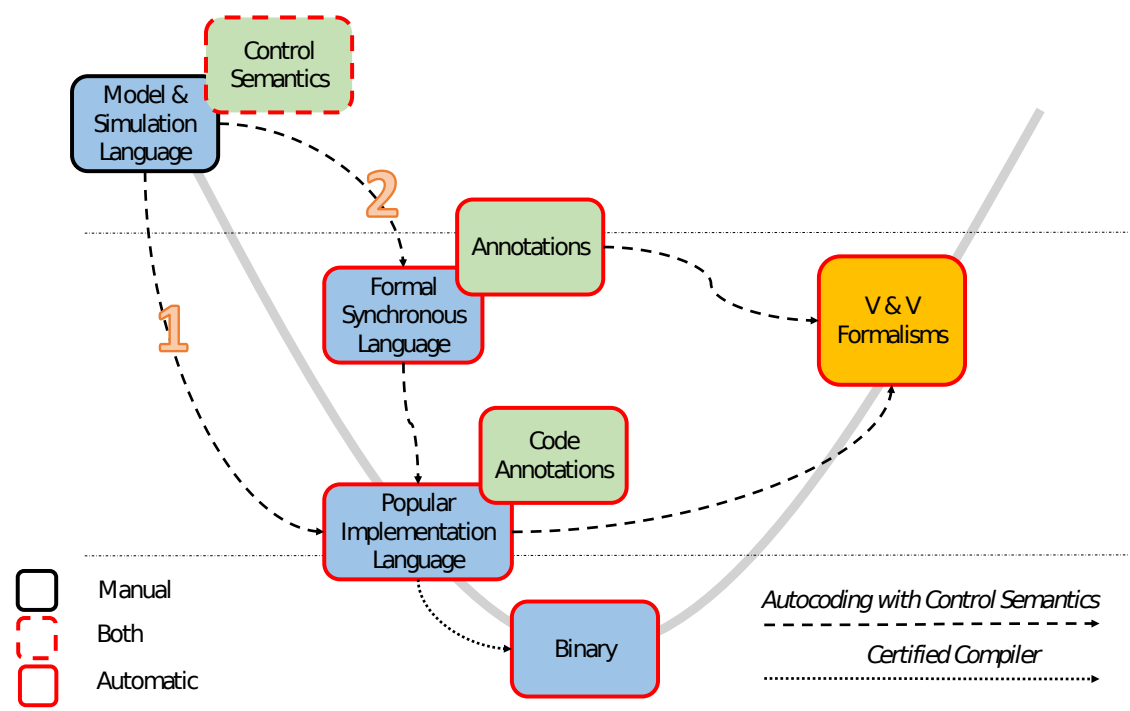

Fig. 1. Automated Credible Autocoding/Compilation Chain for Control Systems

annotations on the code. In the second branch of the framework, the input model is first translated into a formal synchronous dataflow language such as Lustre. At the Lustre level, the control semantics now expressed in a new Lustre specification language, can be analyzed and verified by adapting existing tools such as Kind or the one described in [12],

The first branch is described in more details in Figure 2. It is consists of a series of autocoding steps that translate the control semantics, inserted at the model-level into a set of Hoare logic annotations for the output code. The language used for the input to the framework should be a convenient graphical dataflow modeling language such as Simulink for example. The exact choice for the input language is up to the domain experts' preference and does not affect the utility of the framework as it can be adapted to other modeling languages. Likewise, for the output language, the choice is likely to depend on the preferences of the industry and the certification authority rather than technical reasons arising from the framework. For the prototype described in this paper, the output language was chosen to be $\mathrm{C}$ because of its industrial popularity and the wide availability of static analyzers tailored for $\mathrm{C}$ code.

The set of annotations in the output source code contains both the functional properties inserted by the domain expert and the proofs that can be used to automatically prove these properties. For the analysis of the annotated output, we built a prototype annotation checker that is based on the static analyzer frama-C and the theorem prover PVS. For automating the proof-checking of the annotated output, a set of linear algebra definitions and theories were integrated into the standard NASA PVS library. 


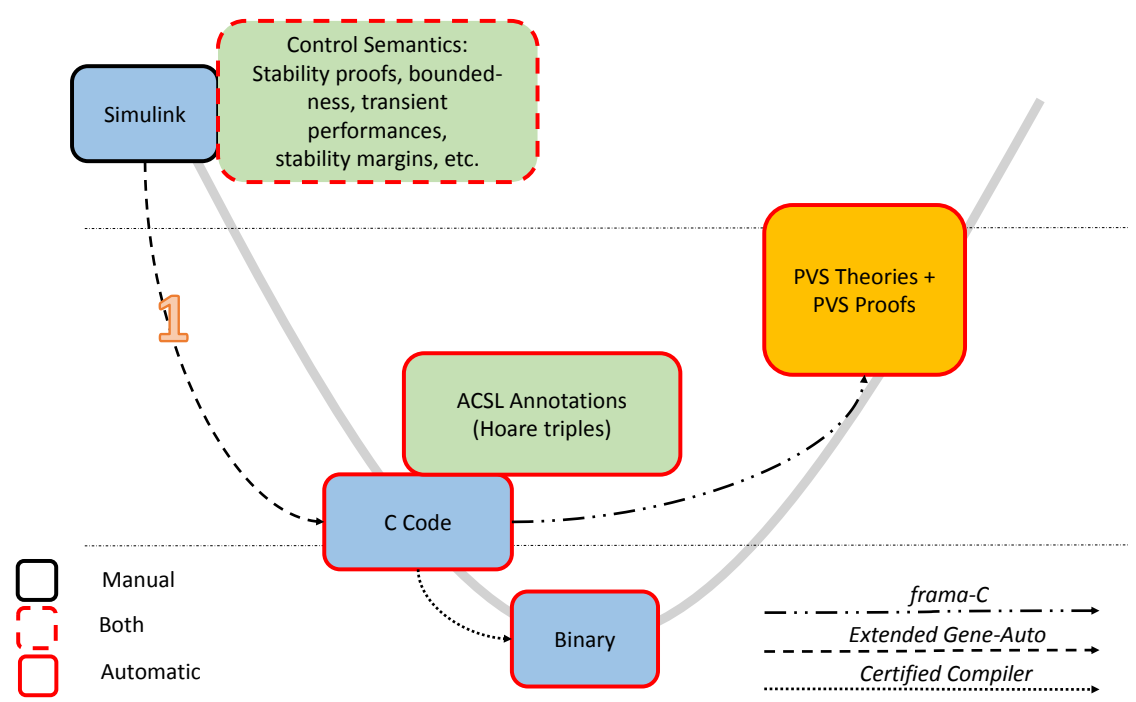

Fig. 2. Autocoding with Control Semantics from Simulink to C

In this paper, the fully automated process from the input model to the verified output is showcased for the property of open loop stability, but the expression of other functional properties on the model are also discussed in this paper. At this point, we restrict the input to only linear controllers with possible saturations in the loop. The running example that we will refer to repeatedly in this paper is the system described by the state-space difference equation in (1). This example has enough complexity to be representative of most controllers used in the industry, and is simple enough such that we can show in this paper, the output annotated code. The system has the states $x \in \mathbb{R}^{2}$, the input $y \in \mathbb{R}$, output $u \in \mathbb{R}$ and the state-transition function parameterized by the 4 matrices in (1).

Example 1.

$$
\begin{gathered}
x_{+}=\left[\begin{array}{cc}
0.4990 & -0.05 \\
0.01 & 1
\end{array}\right] x+\left[\begin{array}{c}
0 \\
0.01
\end{array}\right] y \\
u=\left[\begin{array}{cc}
564.48 & 0
\end{array}\right] x+[1280] y .
\end{gathered}
$$

However note that the framework has been applied to larger systems, which include the Quanser 3-degree-of-freedom Helicopter and an industrial F/A-18 UAV controller system.

\section{Control Semantics}

The set of control semantics that we can express on the model include but is not limited to stability and boundedness. Note that in this paper, the only control semantics of example 1 that we are going to demonstrate through the entire 
autocoding and proof-checking process is the proof of open-loop stability. Nevertheless, we will also describe some other control semantics that can be expressed on the input Simulink model and then translated into C code annotations.

The types of systems in which we can express open-loop stability properties for are not just limited to simple linear systems such as example 1. They also include certain nonlinear systems that can be modeled as linear systems with bounded nonlinearities in the feedback loops.

\subsection{Control System Stability and Boundedness}

A linear control system such as the running example is formally a sextuple consisted of an input alphabet $y \in \mathbb{R}^{m}$, an output alphabet $u \in \mathbb{R}^{k}$, a set of states $x \in \mathbb{R}^{n}$, an initial state $x(0)=x_{0}$, a linear state-transition function $\delta:(x, y) \rightarrow x_{+}$defined by the pair of matrices $\left(A \in \mathbb{R}^{n \times n}, B \in \mathbb{R}^{n \times m}\right)$, and a linear output function $\omega:(x, y) \rightarrow u$ defined by another pair of matrices $\left(C \in \mathbb{R}^{k \times n}, D \in \mathbb{R}^{k \times m}\right)$. In control, we simply express these sextuples using the following state-space formalism

$$
\begin{aligned}
& x_{+}=A x+B y, x(0)=x_{0} \\
& u=C x+D y .
\end{aligned}
$$

There are many inherent nonlinearities in a realistic control system such as timedelays, noise or unmodelled plant dynamics. Additionally, controller components such as safety limiters and anti-windup mechanisms also produce nonlinearities. One tractable way to handle all of these nonlinearities is to abstract them as bounded nonlinear operators. For a more accurate representation of the control systems that are in operation, we also consider a class of nonlinear system that is consisted of a linear system modelled by (2) in feedback interconnections with a set of bounded nonlinear operators. Let the input alphabet $w \in \mathbb{R}^{l}$ be such

that $w=\sigma(u, k)$. Let $B_{w} \in \mathbb{R}^{n \times l}$, we have the following nonlinear state-space system

$$
\begin{aligned}
& x_{+}=A x+B y+B_{w} w, x(0)=x_{0} \\
& u=C x+D y .
\end{aligned}
$$

For systems described by (3), which is inclusive of systems described by (2), it is possible to compute in polynomial time the answer to the quadratic stabilizability problem.

Problem 1. 1. Assume that the input $y$ is bounded i.e. without loss of generality let $\|y\| \leq 1$, and $w$ is a bounded nonlinear operator, does there exist a matrix $P \in \S^{n \times n}, P \succ 0$, such that the quadratic function $q: \rightarrow x^{\mathrm{T}} P x$ is non-increasing along the system trajectories as $k \rightarrow+\infty$ ?

Problem 1 can be reformulated into a linear matrix inequality (LMI) problem. The details of such reformulations are skipped here in this paper as one can refer to, in the system and control literature, a large collection of works on the subject including [14], [13], and [3].

Without proof, here we will state that for bounded $y$ and $w=0$, the following result on an invariant for the system in (2) holds. 
Proposition 2.1 Assume $y^{\mathrm{T}} y \leq 1$. If there exist a $P \succ 0, \alpha>0$, such that

$$
\left[\begin{array}{cc}
A^{\prime \mathrm{T}} P A-(1-\alpha P) & A^{\mathrm{T}} P B \\
B^{\mathrm{T}} P A & B^{\mathrm{T}} P B-\alpha I_{m \times m}
\end{array}\right] \prec 0
$$

then $\left\{x \mid x^{\mathrm{T}} P x \leq 1\right\}$ is an invariant for (2).

The linear matrix inequailty in (4) can be solved for $P \succ 0$ using existing semidefinite programming solvers such as SeDuMi, SDPT3, CSDP, etc.

For $w \neq 0$, we need to first characterize the nonlinearity $w=\sigma(u, t)$. For example, a saturation operator on the output $u$ can be captured in a sector inequality defined by $m_{1}, m_{2}>0$ and $\left(w-m_{1} u\right)^{\mathrm{T}}\left(w-m_{2} u\right)$.

\section{Proposition 2.2}

It is not possible to construct a single algorithm to automate this step in the analysis.

\subsection{Control Semantics in Simulink}

Both boundedness and stability can be expressed using a synchronous observer with inputs $x_{i}, i=1, \ldots, n$, and the boolean-valued function

$$
x \rightarrow \sum_{i, j=1, \ldots, n} x_{i} P_{i j} x_{j} \leq 1 .
$$

This synchronous observer is parameterized by a symmetric matrix $P$ and a multiplier $\mu$. We make an important distinction between two types of ellipsoid observers. One is inductive and the other one is assertive. This distinction originates from the method used to obtain the parameter $P$ and is determined by the memory characteristics of the block's inputs. The former type must have input signals with memories i.e. if the input signals are connected with unit delay blocks. The inductive type expresses an invariant property of the control system loop. Its parameter $P$ is obtained by solving the quadratic stability problem in 1. The assertive type accepts only input signals with no memory i.e. not connected with any unit delay block. Its parameter $P$ is obtained from assertions i.e. assumptions about the sizes of its input signals.

The inductive ellipsoid observer provides both the boundedness of the controller states and the proof of the boundedness itself. The bound on the states can

be extracted from the parameter $P$ by computing the interval $\left[-\frac{1}{\sqrt{\sigma_{i}(P)}}, \frac{1}{\sqrt{\sigma_{i}(P)}}\right]$, where $\sigma_{i}(P)$ is the $i$-th singular value of $P$. The proof comes from the fact that the parameter $P$ is the answer to the quadratic stability problem hence the following holds true.

Fact 2.3 $\forall x \in \mathbb{R}^{n}$, if $y$ is bounded and $P$ is the solution to the quadratic stability problem in 1 , then $(A x+B y)^{\mathrm{T}} P(A x+B y) \leq x^{\mathrm{T}} P x$. 
Fact 2.3 is true by the construction of $P$.

For expressing the ellipsoid observers on the Simulink model, we constructed a custom S block denoted as Ellipsoid to represent the ellipsoid observer. Additionally, for expressing the operational semantics of the plant, we constructed a custom S block denoted as Plant. Its semantics is similar to Simulink's discretetime state-space block with two key differences. One is that the input to the Plant block contains both the input and output of the plant. The other is that the output from the Plant block are the internal states of the plant. Note that the outputs from the Plant block represent variables with memories so they can also be inputs to an inductive ellipsoid observer. Other properties can also be expressed such as non-expansivity from the dissipativity framework. The NonExpansivity block, when connected with the appropriate inputs and outputs, can be used to express a variety of performance measures such as the $H_{\infty}$ characteristic of the system or the closed-loop vector margin of the control system. An example of such usage is shown in figure 3 where the closed-loop vector margin of a constant gain controller is expressed using a combination of the Plant block and the Non-Expansivity block.

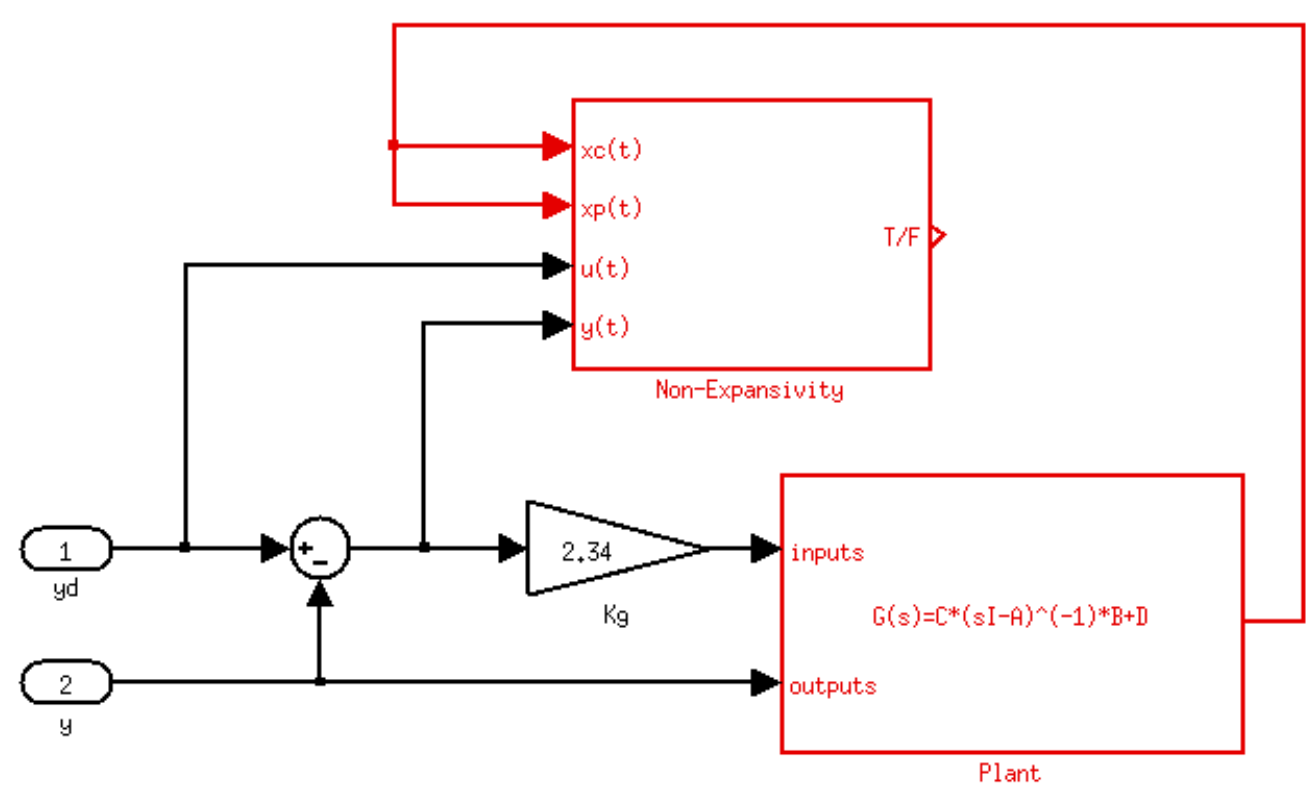

Fig. 3. Expressing Vector Margin of the Closed-Loop System

In this paper, we focus on the current fully-automated treatment of the openloop stability properties, hence we will not consider the semantics displayed in figure (3) beyond the description here. 
For the running example, we have a Simulink model connected with with two synchronous observers. The observers are displayed in red for clarity's purpose. In the Simulink model, we introduce an error into the control system by flipping the sign of gain block $A 11$ in figure 4 . This error will be referred to again in the next section of paper. We made the following assumption for the quantity

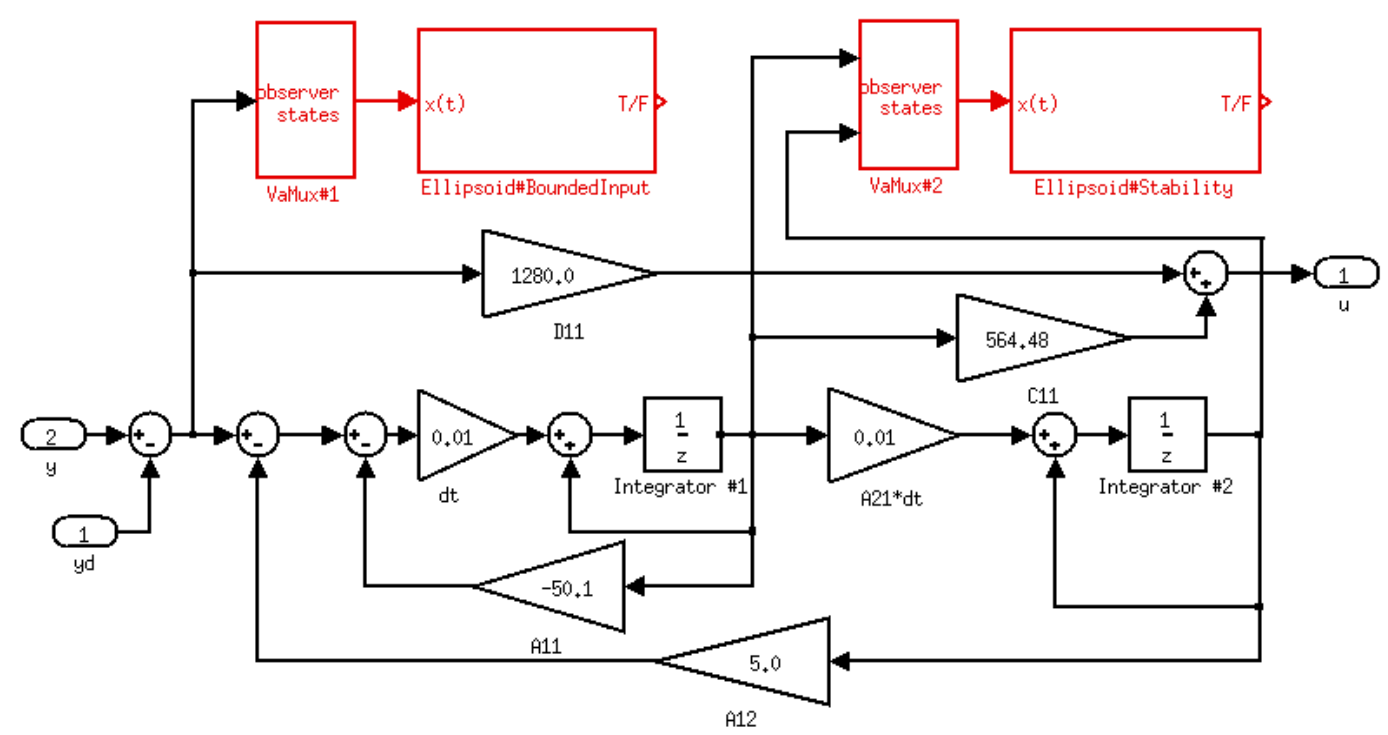

Fig. 4. Running Example with Synchronous Observers

$y-y_{d}$

$$
\left\|y-y_{d}\right\| \leq 0.5
$$

which is expressed in figure 4 by the Ellipsoid block BoundedInput with the parameters $P=0.5$ and multiplier $\mu=0.0009$. The stability proof is expressed in figure 4 by the Ellipsoid block Stability with the parameters $P=$ $\left[\begin{array}{cc}6.742 \times 10^{-4} & 4.28 \times 10^{-5} \\ 4.28 \times 10^{-5} & 2.4651 \times 10^{-3}\end{array}\right]$ and $\mu=0.9991$. The observer blocks in figure 4 are connected to the model using the VaMux block. The role of the VaMux block is to concatenate a set of scalar signal inputs into a single vector output. This special block was constructed because the Ellipsoid observer block can accept only a single vector input.

\subsection{Control semantics at the level of the $\mathrm{C}$ code}

For the specific problem of open loop stability, the expressiveness needed at the $\mathrm{C}$ code level is twofold. On the one hand, a means of expressing that a vector composed of program variables is a member of an ellipsoid is required. This 
entails a number of underlying linear algebra concepts. On the other hand, a eans of providing the static analysis tools with indications on how to proceed with the proof of correctness.

ACSL, the ANSI/ISO C Specification Language, is an annotation language for $\mathrm{C}$ [2].It is expressive enough, and its associated verification tool, Frama$\mathrm{C}$, offers a wide variety of backend provers which can be used to establish the correctness of the annotated code.

Linear Algebra in ACSL A library of ACSL symbols has been developed to express concepts and properties pertaining to linear algebra. In particular, types have been defined for matrices and vectors, and predicates expressing that a vector of variables is a member of the ellipsoid $\mathcal{E}_{P}$ defined by $\{x \in$ $\left.\mathbb{R}^{n}: x^{\mathrm{T}} P x \leq 1\right\}$, or the ellipsoid $\mathcal{G}_{X}$ defined by $\left\{x \in \mathbb{R}^{n}:\left[\begin{array}{ll}1 & x^{\mathrm{T}} \\ x & X\end{array}\right] \geq 0\right\}$. For example, expressing that the vector composed of program variables $v_{1}$ and $v_{2}$ is in the set $\mathcal{E}_{P}$ where $P=\left(\begin{array}{ll}1.53 & 10.0 \\ 10.0 & 507\end{array}\right)$, can be done with the following ACSL code:

I*@ logic matrix P = mat_of_2x2_scalar $(1.53,10.0,10.0,507)$;

Q assert in_ellipsoid(P,vect_of_2_scalar $\left.\left(\mathrm{v}_{-} 1, \mathrm{v}_{-} 2\right)\right)$; */

The stability of ellipsoid $\mathcal{E}_{P}$ throughout any program execution can be expressed by the following loop invariant:

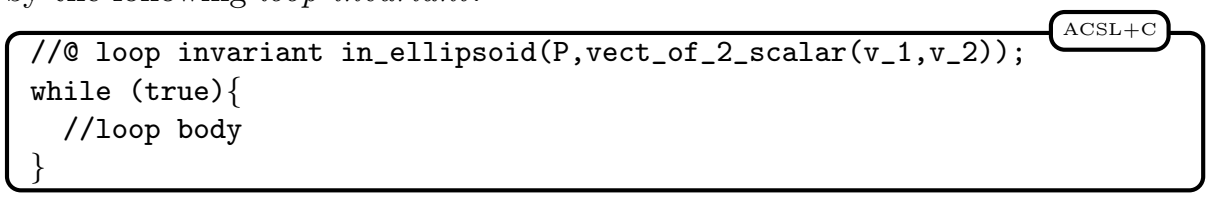

In terms of expressiveness, this latter annotation is all that is required to express open loop stability of a linear controller. However, in order to facilitate the proof, intermediate annotations are added within the loop to propagate the ellipsoid through the different variable assignments, as suggested in [5] and expanded on in section 3. For this reason, a loop body instruction can be annotated with a local contract, like so:

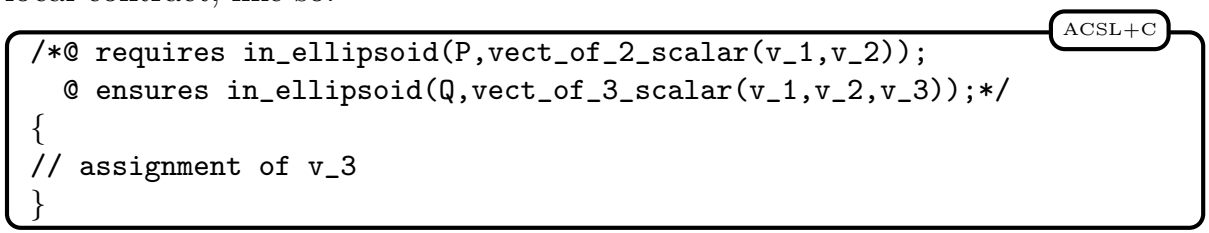

Including proof elements An extension to ACSL, as well as a plugin to Frama-C have been developed. They make it possible to indicate the proof steps needed to show the correctness of a contract, by adding extra annotations. For example, the following syntax: 


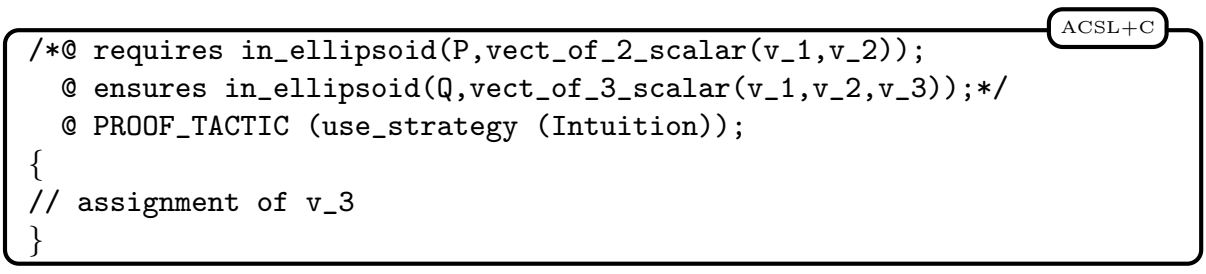

signals Frama-C to use the strategy 'Intuition' to prove the correctness of the local contract considered. Section 4 expands on this topic.

\subsection{Control semantics in PVS}

Through a process described in section 4, verifying the correctness of the annotated $\mathrm{C}$ code is done with the help of the interactive theorem prover PVS. This type of prover normally rely on a human in the loop to provide the basic steps required to prove a theorem. In order to reason about control systems, linear algebra theories have been developed. General properties of vectors and matrices, as well as theorems specific to this endeavor have been written and proven manually within the PVS environment.

Basic types and theories Introduced in [7] and available at [URL] as part of the larger NASA PVS library, the PVS linear algebra library enables one to reason about matrix and vector quantities, by defining relevant types, operators and predicates, and proving major properties. To name a few:

- A vector type.

- A matrix type, along with all operations relative to the algebra of matrices.

- Various matrix subtypes such as square, symmetric and positive definite matrices.

- Block matrices

- Determinants

- High level results such as the link between Schur's complement and positive definiteness

Theorems specific to control theory In [7], a theorem was introduced, named the ellipsoid theorem. A stronger version of this theorem, along with a couple other useful results in proving open loop stability of a controller, have been added to the library. The following theorem

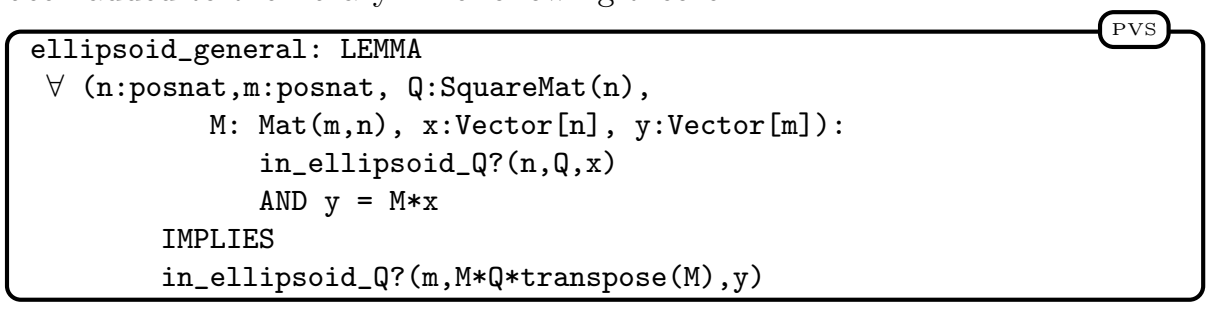


expresses in the PVS syntax how a generic ellipsoid $\mathcal{G}_{Q}$ is transformed into $\mathcal{G}_{M Q M^{T}}$ by the linear mapping $x \mapsto M x$. This next theorem:

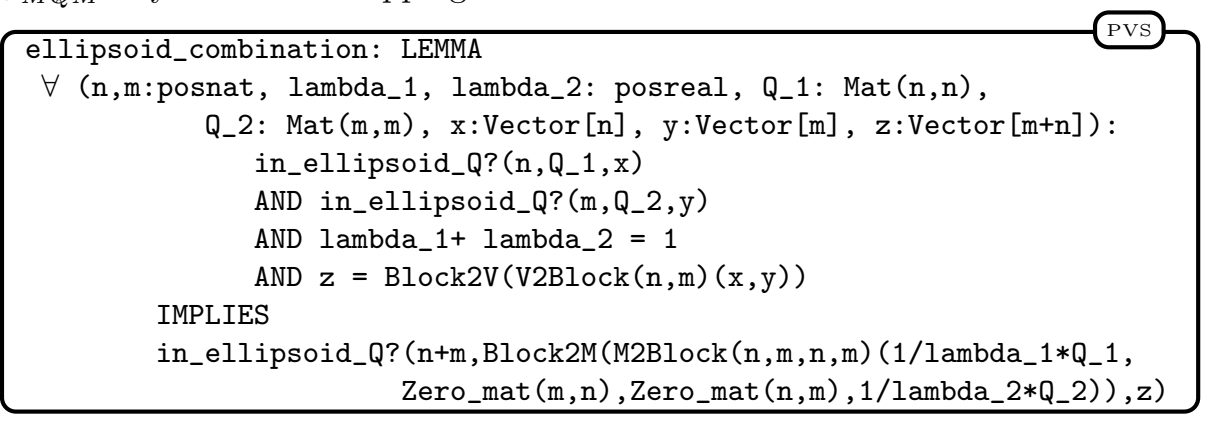

expresses how, given 2 vectors $x$ and $y$ in 2 ellipsoids $\mathcal{G}_{Q_{1}}$ and $\mathcal{G}_{Q_{2}}$, and a multiplier $\mu$, it can always be said that $\left(\begin{array}{l}x \\ y\end{array}\right) \in \mathcal{G}_{Q}$, where $Q=\left(\begin{array}{cc}\frac{Q_{1}}{\mu} & 0 \\ 0 & \frac{Q_{2}}{1-\mu}\end{array}\right)$

These 2 theorems are used heavily in section 4 to prove the correctness of a block.

\section{Autocoding with Control Semantics}

We have so far defined the annotations blocks to express control semantics at the model level. In this Section, we describe in more details, the prototype autocoder that we built to transform the set of control semantics, defined in Section (2), into analyzable ACSL annotations on the code. The prototype is based on GeneAuto, which is an existing industrial-capable automatic code generator for realtime embedded systems[9].

\subsection{Introduction to Gene-Auto}

Gene-Auto's translation architecture is consisted of a sequences of independent model transformation stages. This classical, modular approach to code generator design has the advantage of allowing relatively easy insertion of additional transformation and formal analysis stages such as the annotations generator in our prototype. Figure 5 has an overview of Gene-Auto's transformation pipeline. The process goes through two layers of intermediate languages. The first one, called the GASystemModel, is a dataflow language semantically similar to the discrete subset of the Simulink formalism. The input Simulink model, after being imported, is first transformed into the system model. The system model, which is expressed in the GASystemModel language, is then transformed into the code model. The code model is in the GACodeModel language representation, which is semantically similar to imperative programming languages such as $\mathrm{C}$ or Ada. The key translational stages from the input model to the output code are the importer, the pre-proccessor, the block sequencer, the typer, the GACodeModel 
generator, and finally the printer. For our prototype, we have recycled much of the translational stages up to the GACodeModel generator. From this point on, the prototype tool branch off from the original pipeline until the printer stage, in which much of that was also recycled.

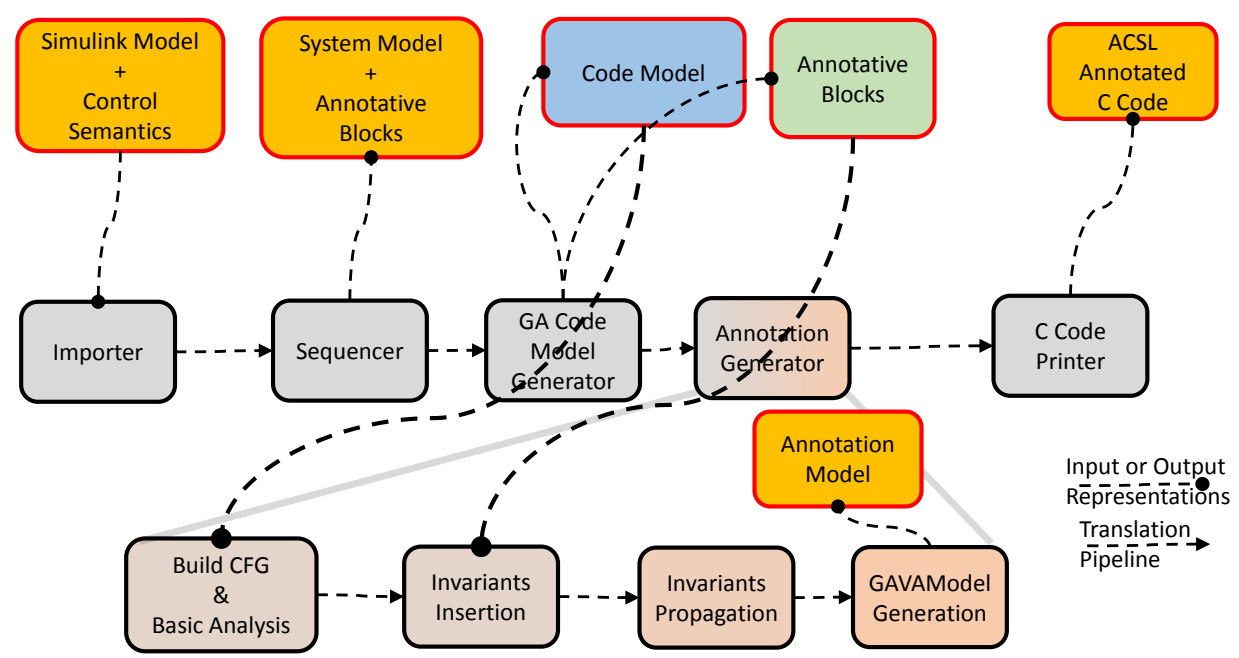

Fig. 5. Gene-Auto Translation Pipeline/Modifications

\subsection{Annotations Generation}

The control semantics are also first transformed into a GASystemModel representation. This transformation step is unaltered from the original Gene-Auto as the formalisms to express the control semantics and the controller model on the Simulink level are very similar. In the GACodeModel generation stage, the system blocks that express the control semantics are not transformed into a GACodeModel representation. Instead, they are imported into the annotations generation module. The annotations generation module is initiated after the code model has been constructed from the system model. The module is responsible for the insertion of the control semantics blocks onto the code model and then transforming them into a GACodeModel Specification Language representation called the GAVAModel. The code model with the control semantics expressed in GAVAModel becomes the output of the annotations generator. This new representation of code and properties is dubbed as the annotation model.

The GAVAModel was added specifically for expressing the control properties and proofs. However it is based the specification language on ACSL[2] so it 
can be used to express other first-order properties about the generated code. A summary of its elements can be seen in Figure 6.

Following Gene-Auto's modular transformation architecture, the annotations generator is added as an independent module within the GACodeModel generation module. The pipeline for the annotation generation module is also shown in Figure 5. The major stages in converting the annotative system blocks in Figure 4 to the annotation model representation are the following:

1. Importing. Convert the code model for into a control flow graph for analysis.

2. Analysis. Basic analysis of the code model like unrolling of finite loops, constant propagation, and affine analysis. All informations obtained about the code model during this step is stored in the control flow graph.

3. Plant block analysis and insertion. Process the Plant block, and inserted it as an affine transformation object onto the control flow graph. If there is no plant, this step is skipped altogether.

4. Observers analysis and insertion. The Ellipsoid observers are typed based on their inputs and then inserted onto the control flow graph as either assertive invariants or inductive ones.

5. Invariant propagation. Additional ellipsoids are generated from the inserted observers using basic Hoare logic with the two additions: the AffineEllipsoid and the SProcedure rules.

6. GAVAModel generation. Convert all the invariants to GAVAModel and insert them as annotations onto the code model. This creates the annotation model.

Here we will skip the details about stages 1 to 2 as they were constructed using standard existing algorithms. We will also skip any details of stage 3 as in this paper, as there is no Plant block in the running example. While information about the plant is critical to expressing closed-loop properties such as performance margins, the scope of this paper is focused mostly on the open-loop stability properties.

\subsection{Observers analysis and Insertion}

Here we will give a short description of the ellipsoid typing and insertion process. The open-loop control semantics are structured in such way that there is one inductive ellipsoid observer on the model with several other assertive ellipsoid observers on the inputs.

Typing of the Ellipsoids The typing algorithm traces the input ports of the block to their corresponding variables and then checks if all of the variables are linked directly to any port from an Unit Delay blocks or an output port on a Plant block. Since this algorithm operates on the GASystemModel level, the typing is done right before the transformation of the Ellipsoid blocks to ellipsoid invariants. 


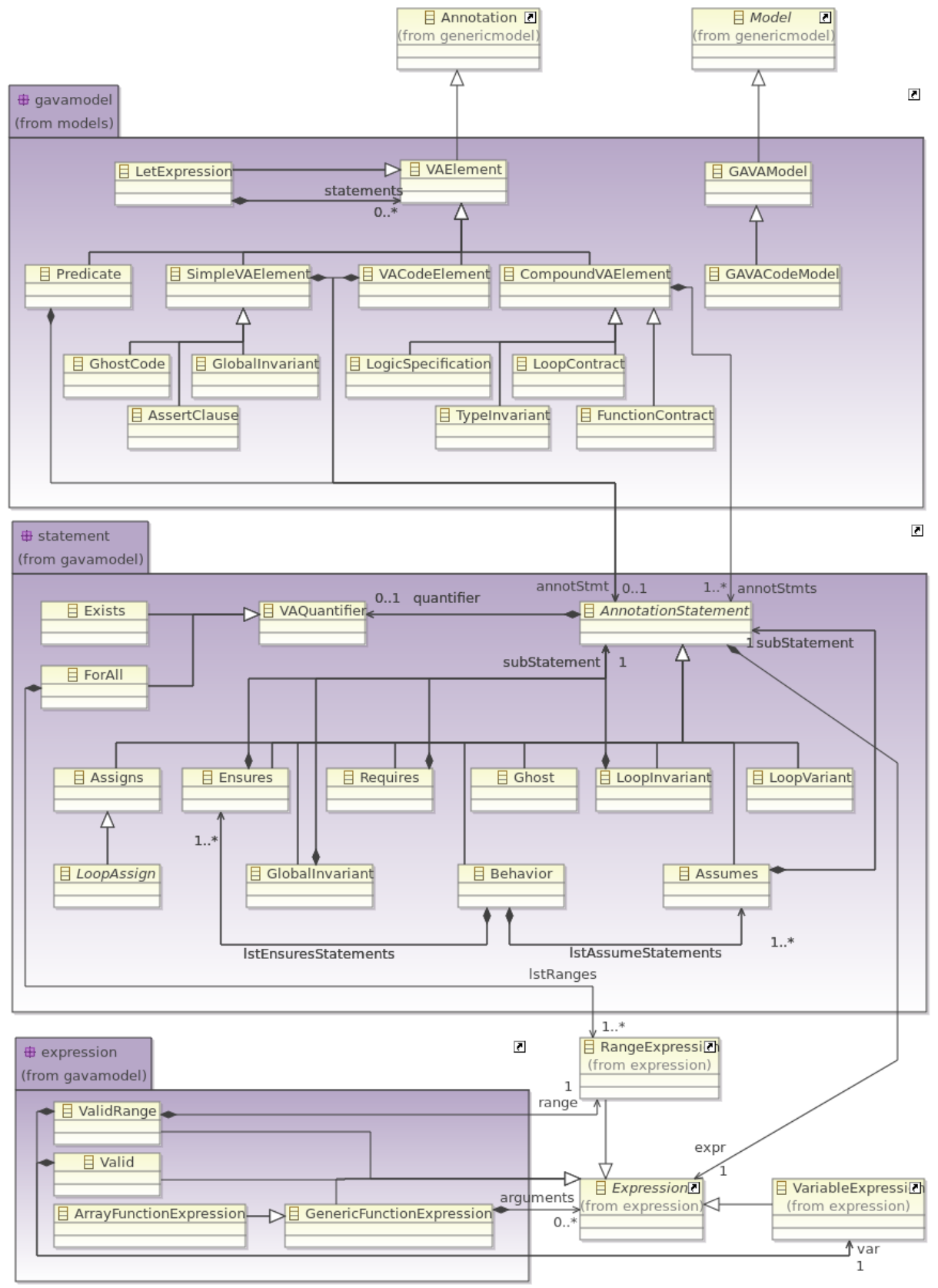

Fig. 6. Specification Language for the Gene-Auto CodeModel 
After the conversion, the equivalent $Q$-form ${ }^{1}$ is computed for each ellipsoid invariant. This conversion is necessary as all subsequent transformation of the ellipsoid invariants are done in the $Q$-form due to the possibility of a degenerate ellipsoid. A degenerate ellipsoid can be visualized in two dimensions as a line segment.

Insertion For the assertive ellipsoids, the insertion location is computed using algorithm 1. The function LHS returns the left hand side of an assignment statement. The variable $\mathrm{G}$ is the control flow graph of the code model. Each node of the control flow graph contains a statement. Simply put, the algorithm founds the first assignment statement where the ellipsoid's state variable is be assigned to and inserts the ellipsoid invariant into the statement's list of post-conditions. For the running example, there is a single assertive ellipsoid invariant on the

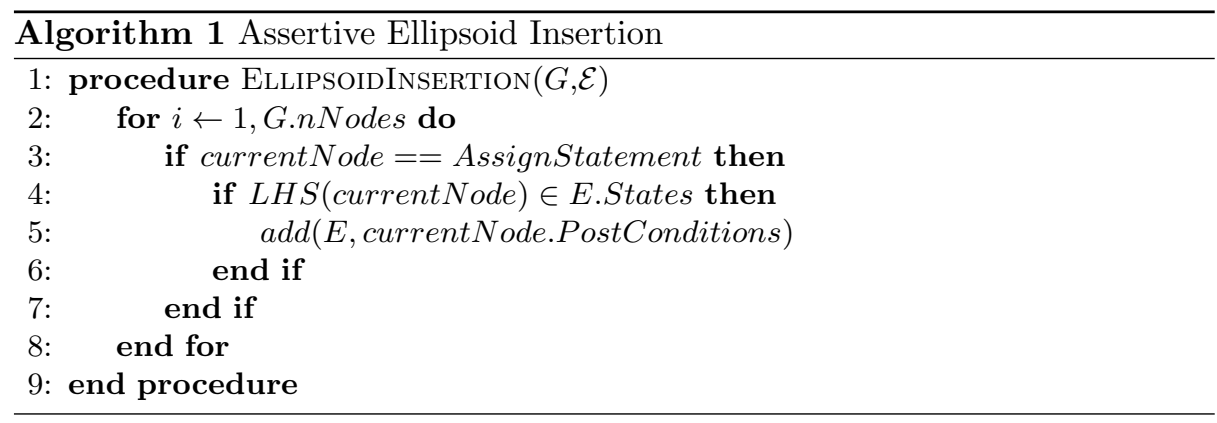

state $\operatorname{Sum} 4=y-y_{d}$ and that is inserted into the code as a post-condition of the line of code that assigns the quantity $y-y d$ to the variable Sum4. This is shown in the autocoded output in Figure 7. The assertive ellipsoid is defined by the matrix variable QMat_11 and is expressed using the function in_ellipsoid $\mathbf{Q}^{2}$. The assumes keyword in ACSL means the formula is an assertion rather than a property to be checked.

The insertion of an inductive ellipsoid invariant is handled differently. The inductive ellipsoid is inserted as pre and post-conditions respectively at the beginning and end of a function body. It is also inserted as a pre and post-conditions on the function itself. The function body is obtained by searching for the function that is linked with all the states of the ellipsoid invariant. If the ellipsoid invariant is linked to more than one function then the search goes up the function call hierarchy until it founds a function that is linked with all the states of the ellipsoid invariant. Figure 8 shows a portion of the autocoded output of the running example. The first three ACSL annotations in Figure 8 defines the matrix

${ }^{1}$ If matrix $P^{-1}$ exists and let $Q=P^{-1}$, then $x^{\mathrm{T}} P x \leq 1$ is equivalent to $\left[\begin{array}{ll}1 & x^{\mathrm{T}} \\ x & Q\end{array}\right] \geq 0$ which is the $Q$-form

2 The function in_ellipsoidQ defines the ellipsoid in the $Q$-form 


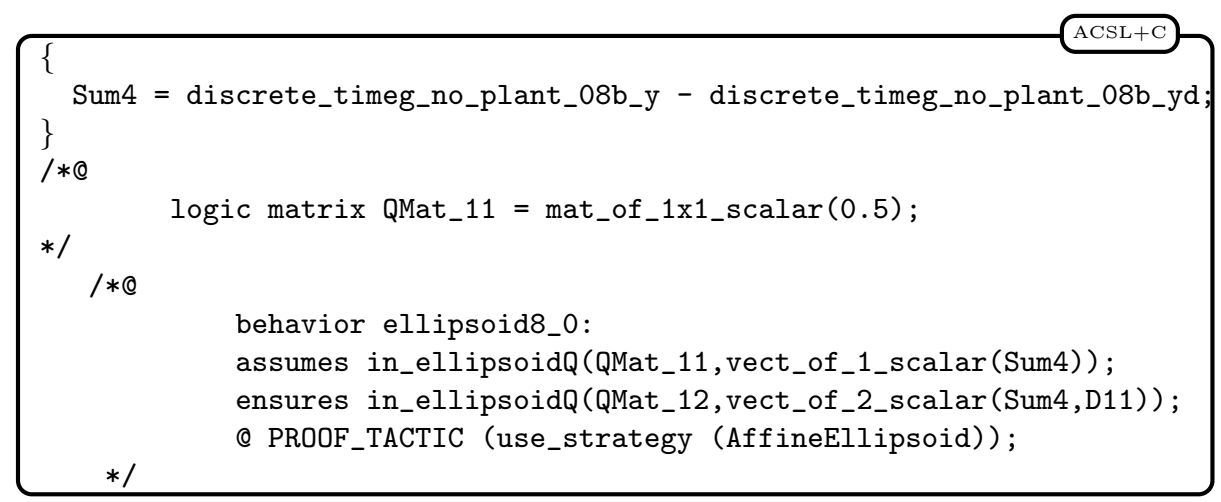

Fig. 7. Assertive Ellipsoid Invariant $\rightarrow$ Assumption in ACSL

variables QMat_0, QMat_1 and QMat_2. All three matrix variables parameterize the same ellipsoid as the one obtained from the stability analysis and inserted into the Simulink model as the Ellipsoid\#Stability observer. Using the ACSL keywords requires and ensures, we can express pre and post-conditions for lines of code as well as functions. The fourth ACSL annotation in Figureprepost expresses the inserted ellipsoid pre and post-conditions for the state-transition function of the controller: discrete_timeg_no_plant_08b_compute. The last ACSL annotation in Figureprepost contains a copy of the pre-condition from the fourth annotation. This is the inserted ellipsoid pre-condition for the beginning of the function body.

\subsection{Invariant Propagation}

The manual forward propagation of ellipsoid invariants was described in [5]. Here we will describe the automatic version of the same process as it is implemented in the prototype. First we give a brief introduction of Hoare logic followed by a description of a targeted extension of Hoare logic to ellipsoid invariants in linear systems. Finally we demonstrate this process on the running example.

Hoare Logic Hoare Logic is a formal proof system that is used to reason about assertions and properties of a program. The key element in Hoare logic is the Hoare triple, which is consisted of a boolean-valued formula, followed by a line of code and then followed by another boolean-valued formula.

$$
\left\{a_{1}\right\} \mathcal{C}_{1}\left\{a_{2}\right)
$$

The meaning of the Hoare triple in ( 7 ) is as follows: if formula $a_{1}$ is true, then after the execution of the line of $\operatorname{code} \mathcal{C}_{1}$, the formula $a_{2}$ is also true. In addition to the Hoare triple, a set of axioms and inferences rules were constructed to reason about the axiomatic semantics of the entire program. Two examples of such 


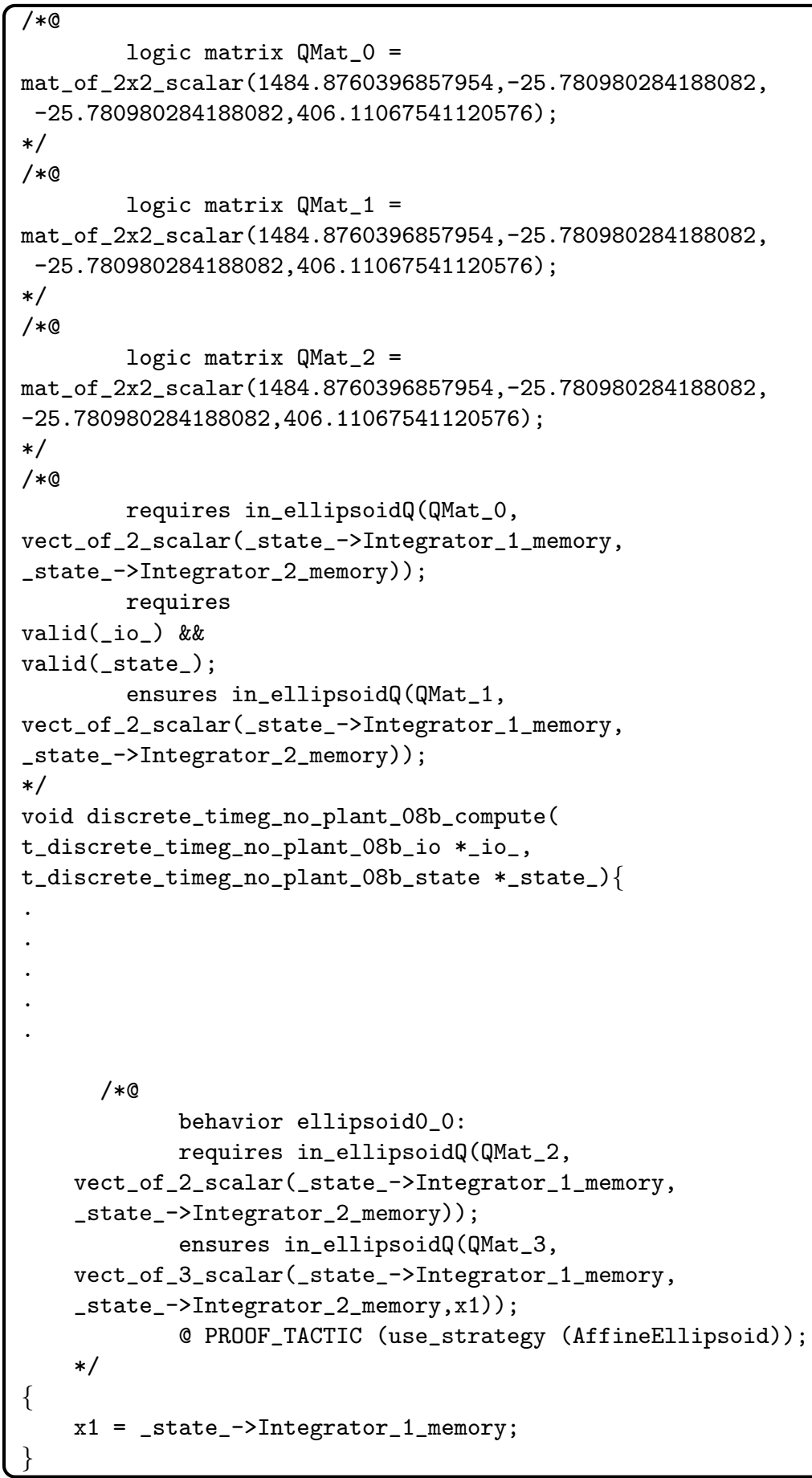$$
\text { . }
$$

Fig. 8. Inductive Ellipsoid Invariant $\rightarrow$ Pre and Post-conditions of the State-Transition Function 
inference rules for a simple imperative language from [8] are listed in Table 1. In order to annotate every lines of the generated code with ellipsoidal pre and postconditions, we also constructed two specialized inference rules for ellipsoids. The first rule is derived from the property of ellipsoid transformation under linear mapping. The second rule is derived from the well-known S-Procedure method in control theory. These two inference rules are sound in the real number domain.

$$
\begin{gathered}
\frac{\left\{\mathcal{E}_{1}\right\} P_{1}\left\{\mathcal{E}_{2}\right\}\left\{\mathcal{E}_{2}\right\} P_{2}\left\{\mathcal{E}_{3}\right\}}{\left\{\mathcal{E}_{1}\right\} P_{1} ; \mathrm{P}_{2}\left\{\mathcal{E}_{3}\right\}}(\text { Composition) } \\
\frac{\vDash\left(\mathcal{E}_{0} \Rightarrow \mathcal{E}_{1}\right)\left\{\mathcal{E}_{1}\right\} P\left\{\mathcal{E}_{2}\right\} \vDash\left(\mathcal{E}_{2} \Rightarrow \mathcal{E}_{n}\right)}{\left\{\mathcal{E}_{0}\right\} P\left\{\mathcal{E}_{n}\right\}} \text { (Consequence) }
\end{gathered}
$$

Table 1. Rules in Hoare Logic

Affine Transformation For the linear propagation of ellipsoids, we define the AffineEllipsoid rule. This rule is applied when the line of code is an assignment statement and has a left-hand expression that is linear. Let the expression $a$ be such that $\llbracket a \rrbracket \vDash L y$, where $y \in \mathbb{R}^{m}$ is vector of program states and $L \in \mathbb{R}^{1 \times m}$. We define the schur form of an ellipsoid using the function $S:(Q, x) \rightarrow\left[\begin{array}{cc}Q & x^{\mathrm{T}} \\ x & 1\end{array}\right]$ and $\mathcal{Q}_{1, x}=\left\{x \mid S\left(Q_{1}, x\right)>0\right\}$. For the Hoare triple,

$$
\left\{\mathcal{Q}_{1}(x)\right\} z:=a\left\{\mathcal{Q}_{2}(x \cup z)\right\}
$$

where $\mathcal{Q}_{n}(x):=\left\{x \mid S\left(Q_{n}, x\right)>0\right\}$, we want to generate $\mathcal{Q}_{2}$ to satify the partial correctness of ( 8 ), we use the following rule.

$$
\overline{\left\{\mathcal{Q}_{n}(x)\right\} z:=a\left\{\mathcal{Q}_{n+1}(x \cup z)\right\}}, Q_{n+1}=\mathcal{F}\left(Q_{n}, \psi(L, y, x), \phi(z, x)\right)
$$

Fig. 9. AffineEllipsoid 
The function $\mathcal{F}$ is defined as follows: given the functions $\psi:(L, y, x) \rightarrow \mathbb{R}^{1 \times n}$ and $\phi:(z, x) \rightarrow \mathbb{Z}$, we have

$$
\begin{aligned}
& \mathcal{F}:\left(Q_{n}, \psi(L, y, x), \phi(z, x)\right) \rightarrow T(\psi(L, y, x), \phi(z, x))^{\mathrm{T}} Q_{n} T(\psi(L, y, x), \phi(z, x)) \\
& T\left(\psi(L, y, x), \phi(z, x)_{i, j}\right):= \begin{cases}1, & 0 \leq i, j \leq n \wedge i=j \wedge i \neq \phi(z, x) \\
0, & 0 \leq i, j \leq n \wedge i \neq j \wedge i \neq \phi(z, x) \\
\psi(y, x)_{1, j}, & i=\phi(z, x) \wedge 0 \leq j \leq n\end{cases} \\
& \psi(L, y, x)_{1, j}:= \begin{cases}L(1, k), & 0 \leq j, k \leq n \wedge x_{j} \in y \wedge y_{k}=x_{j} \\
0, & 0 \leq j \leq n \wedge x_{j} \notin y\end{cases} \\
& \phi(z, x):= \begin{cases}i, & z \in x \wedge z=x_{i} \\
n+1, & z \notin x\end{cases}
\end{aligned}
$$

The ReduceEllipsoid rule is related to the AffineEllipsoid hence they are grouped together. We have the following rule,

$$
\overline{\left\{\mathcal{Q}_{n}(x)\right\} P\left\{\mathcal{Q}_{n+1}\left(\left\{x_{i}\right\} \backslash\{z\}\right)\right\}}, Q_{n+1}=\mathcal{G}\left(Q_{n}, \theta(z, x)\right)
$$

Fig. 10. ReduceEllipsoid

The function $\mathcal{G}$ is defined as the following: given the function $\theta:(z, x) \rightarrow \mathbb{Z}$, we have

$$
T\left(\theta(z, x)_{i, j}\right):=\left\{\begin{array}{c}
\mathcal{G}:\left(Q_{n}, \theta(z, x)\right) \rightarrow T(\theta(z, x))^{\mathrm{T}} Q_{n} T(\theta(z, x)) \\
1,0 \leq i, j \leq n-1 \wedge((i<\theta(z, x) \wedge i=j) \vee(i \geq \theta(z, x) \wedge j=i+1)) \\
0,0 \leq i, j \leq n-1 \wedge((i<\theta(z, x) \wedge i \neq j) \vee(i \geq \theta(z, x) \wedge j \neq i+1)) \\
\theta(z, x):=\left\{i, z=x_{i}\right.
\end{array}\right.
$$

Each line of code is analyzed to determine if it is an affine assignment. If it is an affine assignment, the transformation matrix $L$ is extracted and stored in the control flow graph. For example, if we have $x=y+2 * z$, then the analyzer returns the transformation matrix $L=[1,2]$. Given the pre-condition ellipsoid $\mathcal{Q}_{i}(x)$, the AffineEllipsoid rule only applies when the line of code is $y:=a$ with $\llbracket a \rrbracket=L z \wedge z \subseteq x$. In Figure 11, we have the annotated C output generated by our prototype for example 1 . In the example, The pre-condition is the ellipsoid in the $Q$-form defined by the variable QMat_21, and the ensuing line of code assigns the expression $\mathbf{d t}_{-}+\mathbf{x} \mathbf{1}$ to the variable Sum2. The affine transformation matrix is $L=[1,1]$ and by applying the AffineEllipsoid rule, we have the ellipsoid transformation matrix $T$ defined by

$$
T=\left\{\begin{array}{l}
T_{i j}=1.0,(i \leq 4 \wedge i=j) \vee(i=6 \wedge(j=6 \vee i=6)) \vee(i=5 \wedge j=6) \\
T_{i j}=0.0, \text { otherwise }
\end{array}\right.
$$




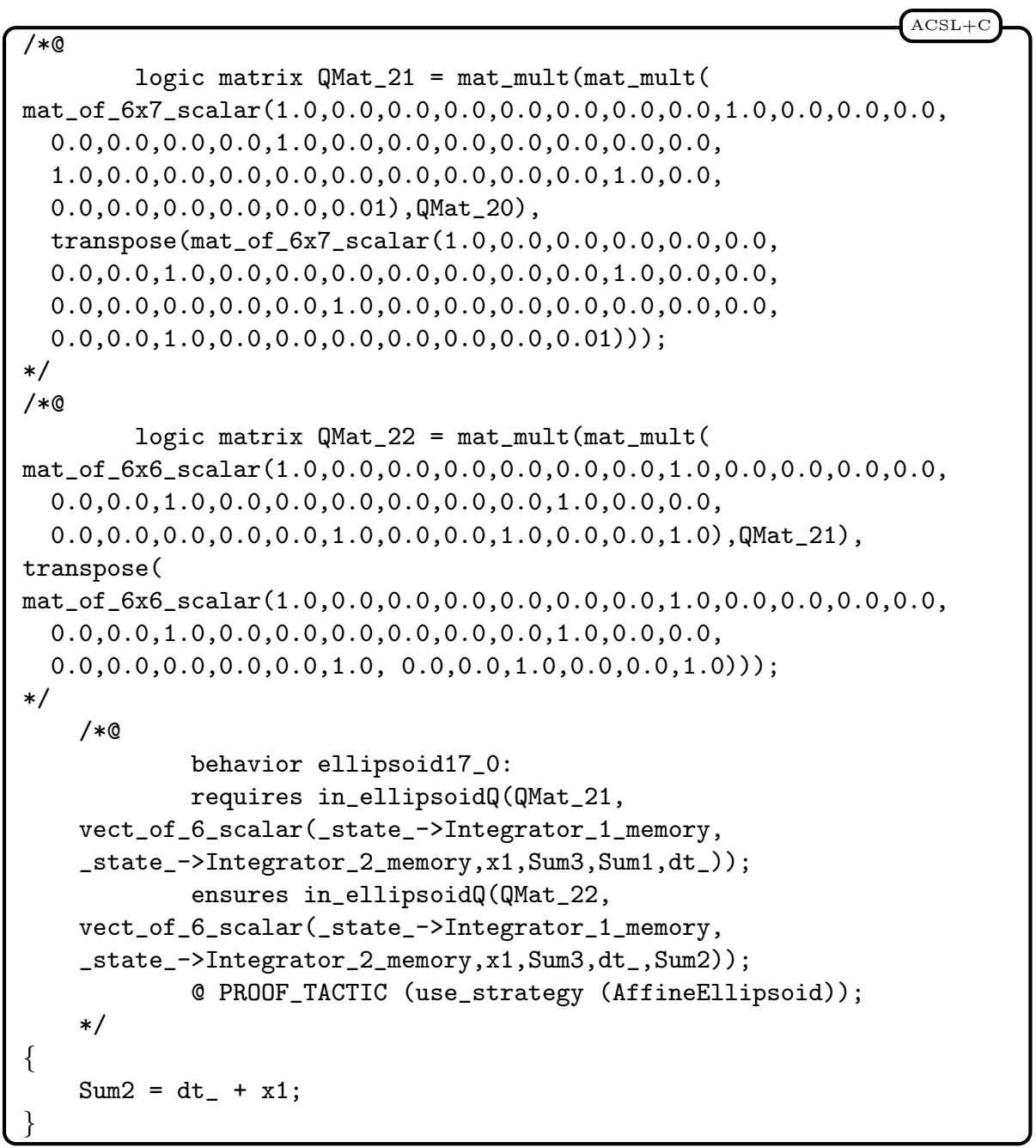

Fig. 11. Application of AffineEllipsoid 
S-Procedure The SProcedure rule is shown in the second ACSL annotation in Figure 12. We will discuss the autocoded output more in detail later on but first we give the definition of the SProcedure rule.

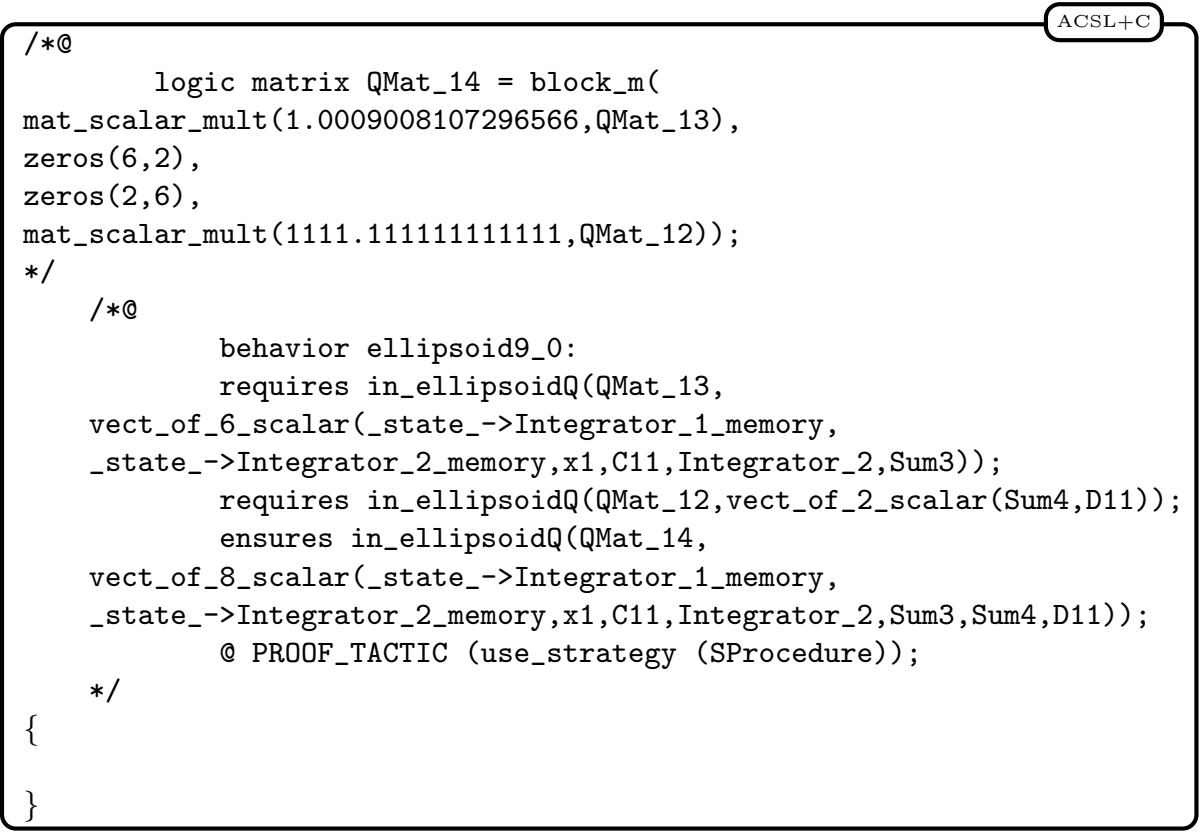

Fig. 12. Application of the SProcedure Rule

The SProcedure rule is activated when two or more ellipsoids can be combined correctly into a single ellipsoid. The definition for SProcedure is displayed in Figure 12. First Let function $\mathcal{H}: \mathbb{R}^{n_{i} \times n_{i}} \rightarrow \mathbb{R}^{N n \times N n}$ be the following: given the function $\operatorname{dim}: R^{n \times n} \rightarrow n$, and the function $\rho: n \in \mathbb{Z}^{+} \rightarrow \sum_{i=1}^{n} \operatorname{dim}\left(Q_{i}\right)$, we have

$$
\mathcal{H}\left(Q_{i}\right)(n, m)= \begin{cases}Q_{i}(n-\rho(i-1), m-\rho(i-1)), & \rho(i-1) \leq n, m \leq \rho(i) \\ 0.0, & \text { otherwise }\end{cases}
$$

The SProcedure rule is:

Given the pre-conditions $\left\{\mathcal{Q}_{i}\left(x_{i}\right)\right\}$ and the code $C$ such that $\llbracket C \rrbracket \vDash(y:=L z)$, the SProcedure rule is activated only when all the following conditions are satisfied.

1. For each $\mathcal{Q}_{i}\left(x_{i}\right)$, the AffineEllipsoid rule does not apply.

2. For the set $\left\{\mathcal{Q}_{i}\left(x_{i}\right)\right\}, i=1, \ldots, N, z \subseteq \bigcup_{i=1}^{N} x_{i}$. 


$$
\begin{gathered}
\overline{\left\{\mathcal{Q}_{1}\left(x_{1}\right) \wedge \mathcal{Q}_{2}\left(x_{2}\right) \wedge \ldots \wedge \mathcal{Q}_{N}\left(x_{N}\right)\right\} \mathbf{S K I P}\left\{\mathcal{Q}_{\mathrm{n}+1}\left(\mathrm{x}_{0} \cup \mathrm{x}_{1} \cup \ldots \cup \mathrm{x}_{\mathrm{n}}\right)\right\}} \\
Q_{n+1}=\sum_{i=1}^{N} \mu_{i} \mathcal{H}\left(Q_{i}\right) .
\end{gathered}
$$

Fig. 13. SProcedure

3. For $\mathcal{Q}_{i}\left(x_{i}\right), i=1, \ldots, N, z \nsubseteq x_{i} \wedge z \cap x_{i} \neq\{\varnothing\}$.

The multipliers are computed beforehand using the S-Procedure to ensure the soundness of the rule in the real number domain. For the running example, we have one ellipsoid defined by the matrix variable QMat_12 as a pre-condition in Figure 12. This ellipsoid is derived from the inserted assertive ellipsoid. The other ellipsiod, also a pre-condition, is defined by the matrix variable QMat_13. This ellipsoid is derived from the inserted inductive ellipsoid. These two ellipsoids are combined to form a new ellipsoid using the SProcedure rule as shown in Figure 12. The new ellipsoid's matrix variable QMat_14, is formulated using the block matrices function block_m.

\subsection{Verification of the Generated Post-condition}

After the invariant propagation step, we obtain a new ellipsoid post-condition defined by the matrix variable PMat_24. It is necessary to check if this new post-condition implies the inserted ellipsoid post-condition defined by the matrix variable QMat_2 in Figure 8. Currently, we can do a numerical verification by using a cholesky decomposition based algorithm with intervals to guarantee a bound on the floating-point computation error. For this particular example, because of the error introduced into the model as mentioned in Section 2, we can see that the new post-condition does not imply the inserted post-condition.

\section{Automatic Verification of Control Semantics}

Once the annotated $\mathrm{C}$ code has been generated, it remains to be proven that the annotations are correct with respect to the code. This is achieved by checking that each of the individual Hoare triple holds. Figure 15 presents an overview of the checking process. First the WP plugin of Frama-C generates verification conditions for each Hoare triple, and discharges the trivial ones with its internal prover QeD. Then the remaining conditions are translated into PVS theorems for further processing, as described in subsection 4.1. It is then necessary to match the types and predicates introduced in ACSL to their equivalent representation in PVS. This is done through theory interpretation [11] and explained in subsection 4.2. Once interpreted, the theorems can be generically proven thanks to PVS strategies, as described in subsection 4.3. In order to automatize these various tasks and integrate our framework within the Frama-C platform, which provides 


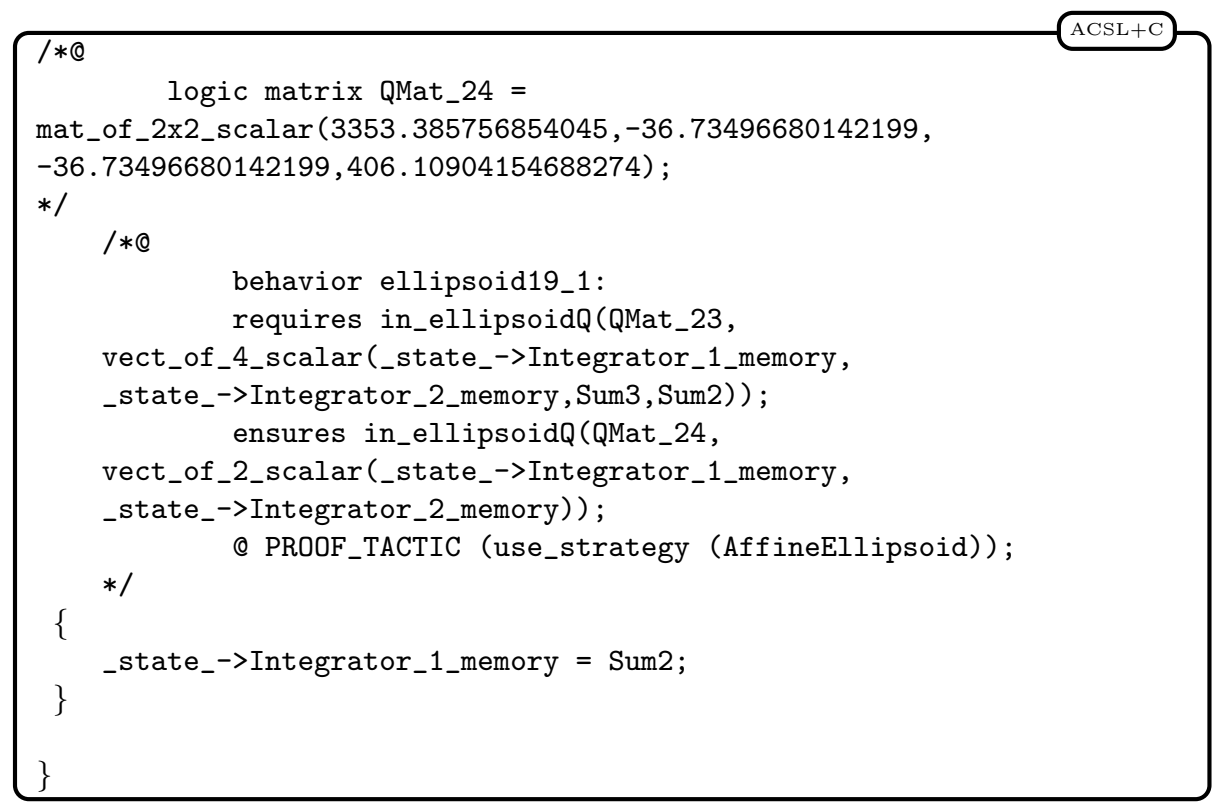

Fig. 14. Generated Post-condition

graphical support to display the status of a verification condition (proved/unproved), a Frama-C plugin named pvs-ellipsoid, described in subsection 4.4, was written. Finally, it must be mentionned here that one last verification condition, quite crucial, does not fall under either AffineEllipsoid of SProcedure strategies. It is discussed in subsection 4.5

\subsection{From C code to PVS theorems}

The autocoder described in the previous Section generates two C functions. One of them is an initialization function, the other implements one execution of the loop that acquires inputs and updates the state variables and the outputs. It is left to the implementer to write the main function combining the two, putting the latter into a loop, and interfacing with sensors and actuators to provide inputs and deliver outputs. Nevertheless, the properties of open loop stability and stateboundedness can be established by solely considering the update function, which this Section will now focus on. The generated function essentially follows the template shown in Figure 16.:

Frama-C is a collaborative platform designed to analyze the source code of software written in $\mathrm{C}$. The WP plugin enables deductive verification of $\mathrm{C}$ programs annotated with ACSL. For each Hoare tripe $\left\{\right.$ pre $\left._{i}\right\}$ inst $_{i}\left\{\right.$ post $\left._{i}\right\}$, it gener- 


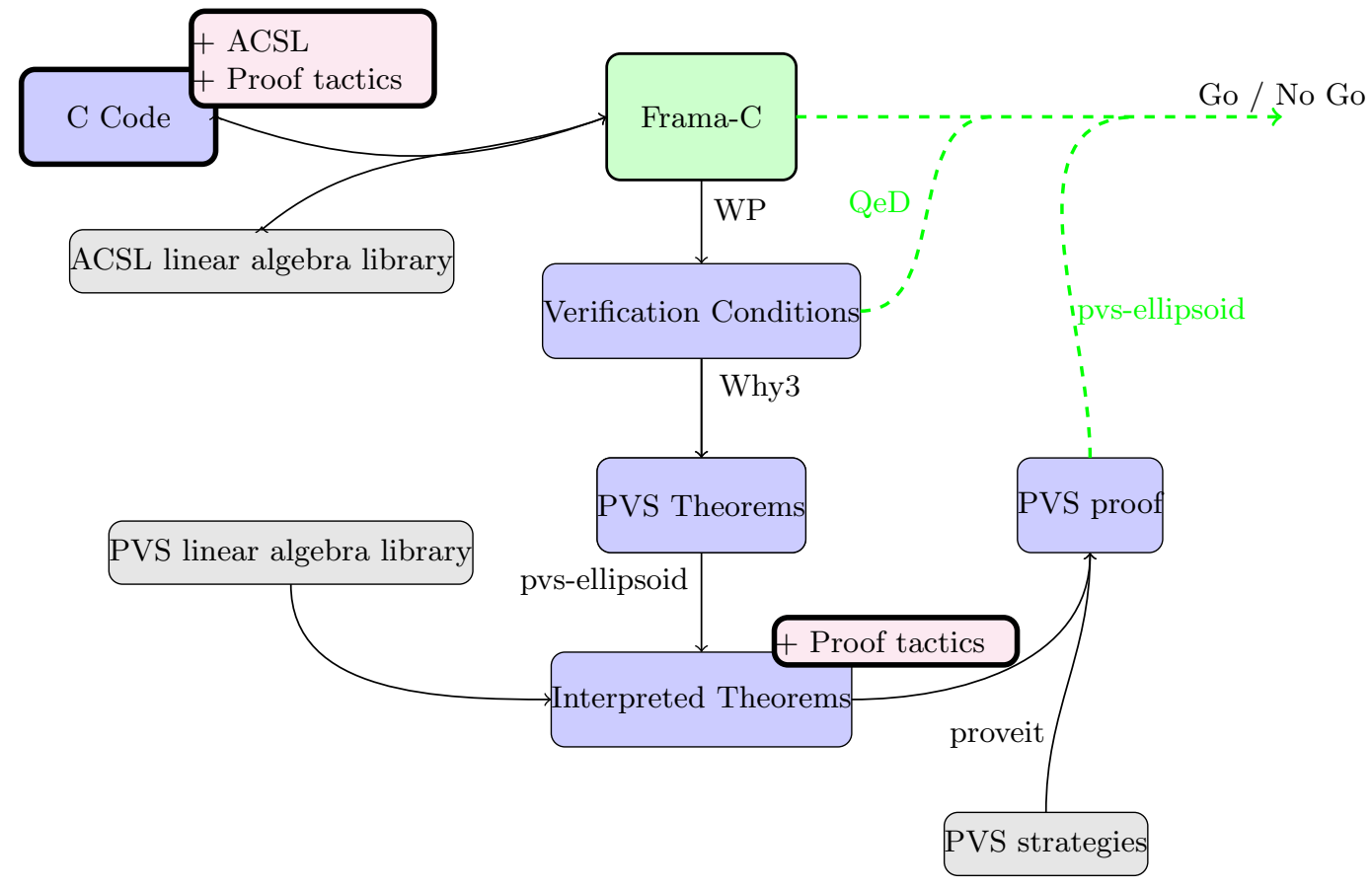

Fig. 15. General view of the automated verification process. The contribution of this Section of the article lies in the domain specific libraries that have been developed at the different layers of description of the code, as well as in the generic proof strategies and the custom Frama-C plugin pvs-ellipsoid

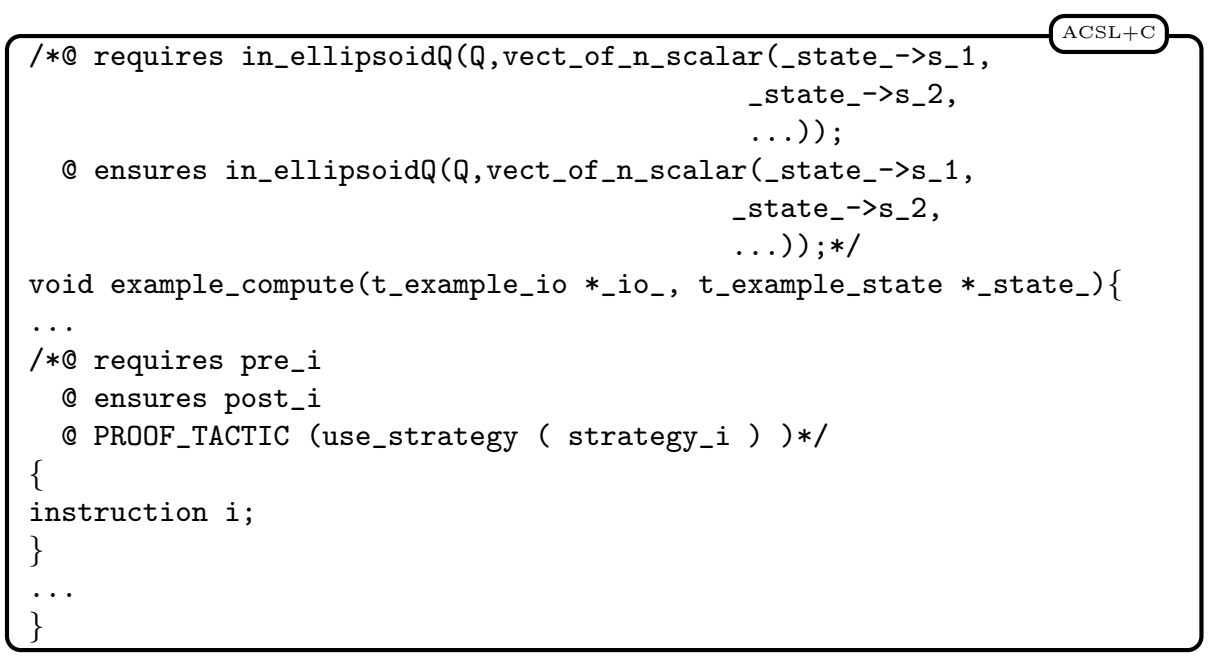

Fig. 16. Template of the generated loop update function 
ates a first order logic formula expressing pre $_{i} \Longrightarrow w p\left(\text { inst }_{i}, \text { post }_{i}\right)^{5}$. Through the Why3 platform, these formulas can be expressed as theorems in PVS, so that, for example, the ACSL/C triple shown in Figure 17, taken directly from our running example, becomes the theorem shown in Figure 18.

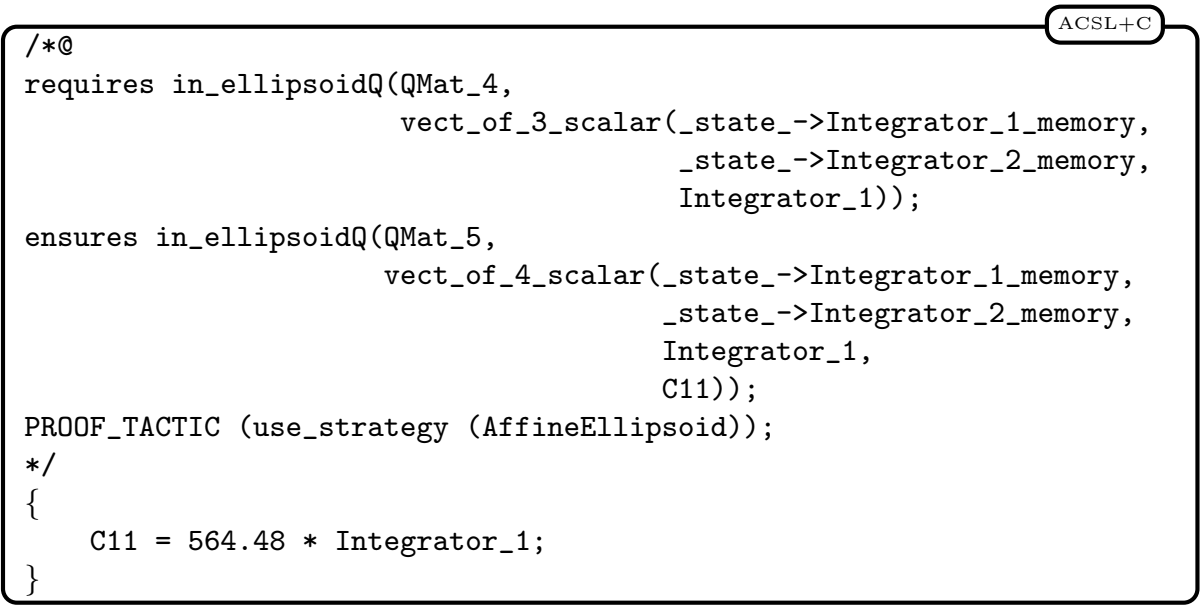

Fig. 17. Typical example of an ACSL Hoare Triple

Note that, for the sake of readability, part of the hypotheses of this theorem, including hypotheses on the nature of variables, as well as hypotheses stemming from Hoare triples present earlier in the code, are ommitted here. Note also that in the translation process, functions like malloc_0 or mflt_1 have appeared. They describe the memory state of the program at different execution points.

\subsection{Theory interpretation}

At the ACSL level, a minimal set of linear algebra symbols have been introduced, along with axioms defining their semantics. Section 2 described a few of them. Each generated PVS theorem is written within a theory that contains a translation 'as is' of these definitions and axioms, along with some constructs specific to handling the semantics of $\mathrm{C}$ programs. For example, the ACSL axiom

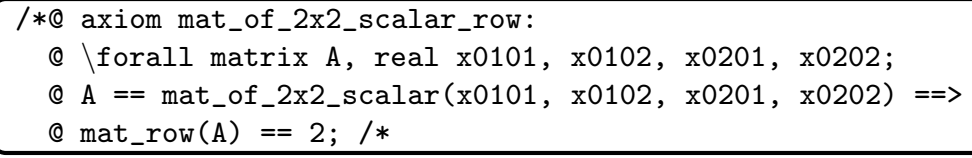

${ }^{5}$ Given a program statement $S$ and a postcondition $Q, w p(S, Q)$ is the weakest precondition on the initial state ensuring that execution of $S$ terminates in a state satisfying $Q$. 


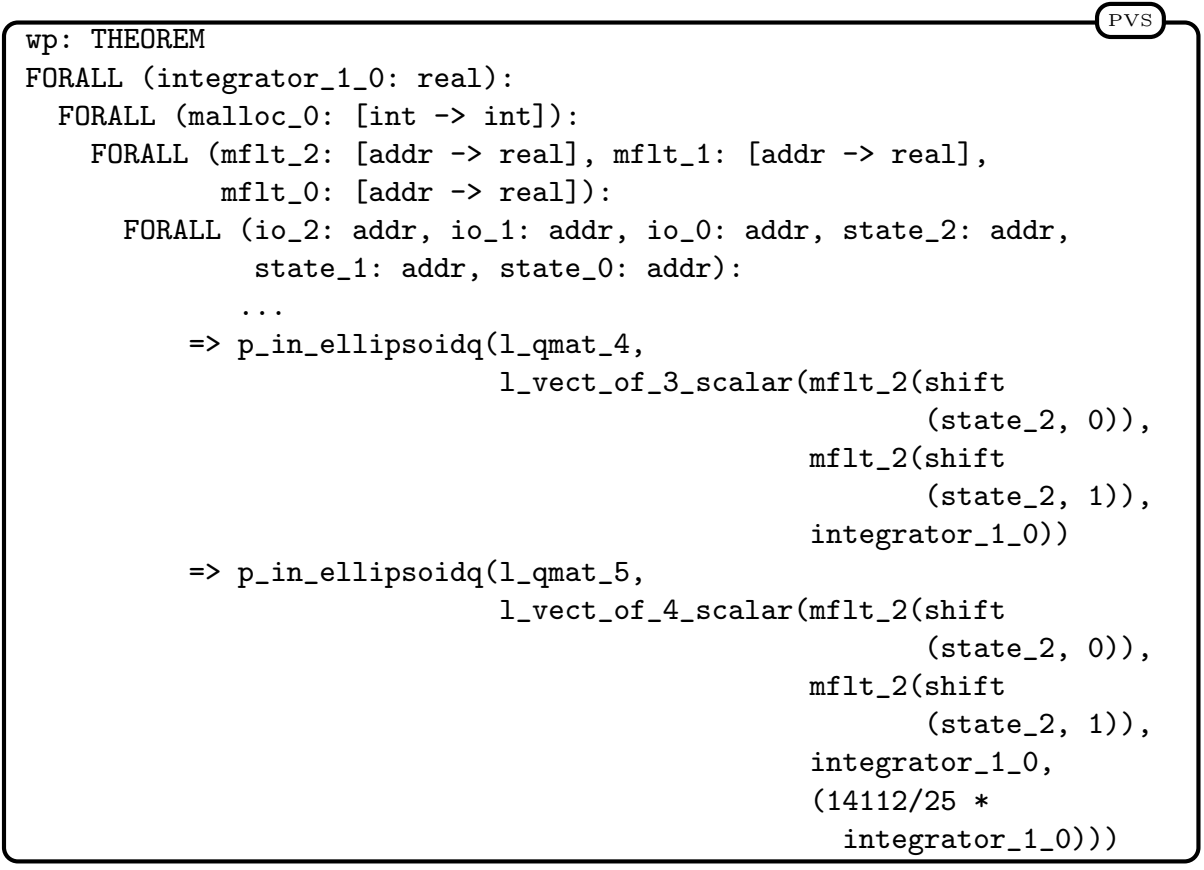

Fig. 18. Exerpt of the pvs translation of the triple shown in Figure 17 
becomes after translation to PVS:

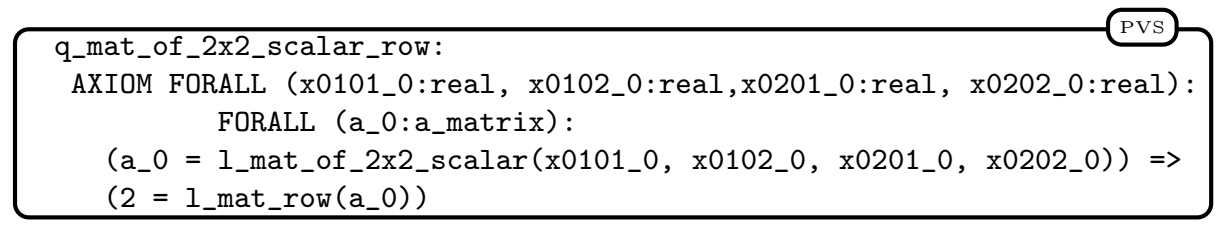

In order to leverage the existing results on matrices and ellipsoids in PVS, theory interpretation is used. It is a logical technique used to relate one axiomatic theory to another. It is used here to map types introduced in ACSL, such as vectors and matrices, to the existing ones in PVS, as well as the operations and predicates on these types. To ensure soundness, PVS requires that what was written as axioms in the ACSL library be reproven in the interpreted formalism.

The interpreted symbols and soundness checks are the same for each proof objective, facilitating the mechanization of the process. Syntactically, a new theory is created in which the theory interpretation is carried out, and the theorem to be proven is automatically rewritten by PVS in terms of its own linear algebra symbols. These manipulations on the generated PVS are carried out by a framaC plugin called pvs-ellipsoid, which will be described further in the following subsection.

\subsection{Generically discharging the proofs in PVS}

Once the theorem is in its interpreted form, all that remains to do is to apply the proper lemma to the proper arguments. Section 3 described two different types of Hoare Triple that can be generated in ACSL. Two pvs strategies were written to handle these possible cases. A pvs proof strategy is a generic function describing a set of basic steps to communicate to the interactive theorem prover in order to facilitate or even fully discharge the proof of a lemma.

The AffineEllipsoid strategy handles any ellipsoid update stemming from a linear assignment of the variables. Recall the following theorem:

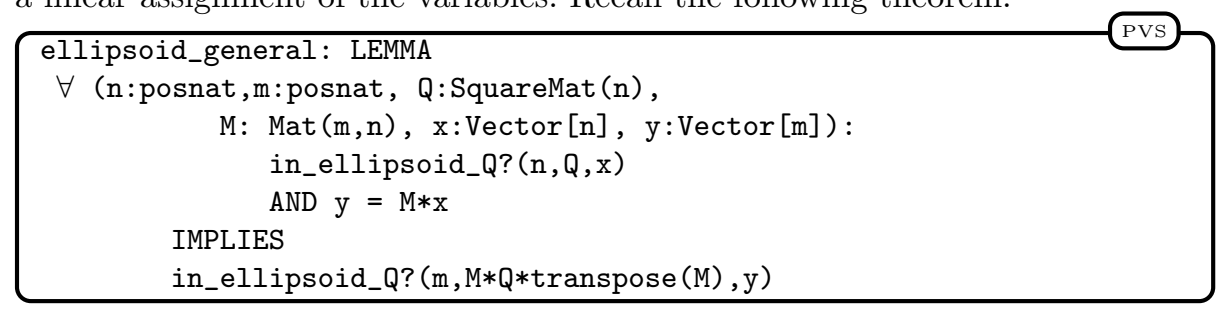

In order to apply it properly, the first step of the strategy consists of parsing through the objectives and hypotheses of the theorem to acquire the name and the dimensions of the relevant variables, as well as isolate the necessary hypotheses. The second step consists of a case splitting on the dimensions of the variable: they are given to the prover in order to complete the main proof, and then established separately using the relevant interpreted axioms. Next it is established that $\mathrm{y}=\mathrm{Mx}$ through a tedious case decomposition and numerous calls to relevant 
interpreted axioms. All the hypotheses are then present for a direct application of the theorem. The difficulties in proof strategy design lie in intercepting and anticipating the typecheck constraints (tccs) that PVS introduces throughout the proof. A third strategy was specifically written to handle them.

The S-Procedure strategy follows a very similar pattern, somewhat simpler since the associated instruction in the Hoare triple is a skip, using the other main theorem presented in Section 2, ellipsoid_combination.

\subsection{The pvs-ellipsoid plugin to Frama-C}

The pvs-ellipsoid plugin to Frama-C automatizes the steps mentionned in the previous subsections. It calls the WP plugin on the provided $\mathrm{C}$ file, then, when QeD fails to prove a step, it creates the PVS theorem for the verification condition through Why3 and modifies the generated code to allow for theory interpretation. It extracts the proof tactic to be used on this specific verification condition, and uses it to signify to the next tool in the chain, proveit, what strategy to use to prove the theorem at hand. proveit is a command line tool that can be called on a pvs file and attempts to prove all the theories in there, possibly using user guidance such as the one just discussed. When the execution of proveit terminates, a report is produced, enabling the plugin to decide whether the verification condition is discharged or not. If it is, a proof file is generated, enabling a replay of proof.

\subsection{The last verification condition}

As mentionned at the beginning of this Section, there is one last verification condition that falls under neither AffineEllipsoid nor S-Procedure category. It is the final post-condition of the main loop function contract, expressing that the state remains in the initial ellipsoid $\mathcal{G}_{P}$. Through a number of transformations, we have a proven chain of assertions which tell us the state is in some ellipsoid $\mathcal{G}_{P}^{\prime}$. The conclusion of the proof lies in the final test $P^{\prime}-P \geq 0$. The current state of the linear algebra library in PVS does not permit to make such a test, however a number of very reliable external tools, like the INTLAB package of the MATLAB software suite, can operate this check. In the case of our framework, the pvs-ellipsoid uses custom code from [12] to ensure positive definiteness of the matrix, with the added benefit of soundness with respect to floating point numbers.

\section{$5 \quad$ Related Works}

The authors would like mention and give thanks to the following related works. We would first like to mention Jerome Ferret and his work on the static analysis of digital filters in [4]. It was this work that springed the connections made between the control-theoretic techniques and software analysis methods in [5]. Furthermore, we would like to mention a parallel work done by Pierre-Loic Garoche 
and Pierre Roux in [12] where policy interation is used to generate and refine ellipsoid invariants. We would like to give thanks to Eric Goubault and Sylvie Putot for the discussions, and mention their work on zonotopal domain for static analyzers [6]. Finally we would like to point out Ursual Martin and her team's work on the Hoare logic for linear systems [1].

\section{Conclusion}

The prototype tools and various examples described in this paper can be found on our svn server. We have demonstrated in this paper a set of prototype tools that is capable of migrating high-level functional properties of control systems down to the code level, and then verifying the correctness of those properties for the code, all in an automatic manner. While the nature of controllers and properties supported is relatively restricted, this effort demonstrate the feasability of a paradigm where domain specific knowledge is leveraged and automatically assists code analysis. This opens the way for numerous directions of research. As the mathematical breadth of theorem provers increase, more and more complex code invariants can theoretically be handled, and thus more and more complex controllers. One major area that should be explored is the integration of the plant model at the level of the code, and the natural compromise that arises between the precision the model and the finesse of the properties that can be proven on the interconnection. This would pave the way for the verification of a wide array of new properties, as most control theory results are expressed on such interconnections between plant and controller. Soundness of the results with respect to floating point computation is another issue that requires attention. We applied the toolchain to a mass-spring-damper system for its open-loop stability property.

\section{Acknowledgements}

The authors would like to thank Pierre Roux for his contribution to the pvsellipsoid plugin, Gilberto Perez and Pablo Ascariz for their invaluable help on the PVS linear algebra library.

This article was prepared under support from the Army Research Office under MURI Award W911NF-11-1-0046, NSF Grant CNS - 1135955 "CPS: Medium: Collaborative Research: Credible Autocoding and Verification of Embedded Software (CrAVES)", FUI 2011 project P, the National Aeronautics and Space Administration under NASA Cooperative Agreement NNL09AA00A, activity 2736, ITEA2 OPES, FNRAE project CAVALE, ANR INS project CAFEIN and ANR ASTRID project VORACE.

\section{References}

1. Rob Arthan, Ursula Martin, and Paulo Oliva. A hoare logic for linear systems. Formal Aspects of Computing, 25(3):345-363, 2013. 
2. Patrick Baudin, Jean-Christophe Filliâtre, Claude Marché, Benjamin Monate, Yannick Moy, and Virgile Prevosto. ACSL: ANSI/ISO C Specification Language, 2008. http://frama-c.cea.fr/acsl.html.

3. S. Boyd, L. El Ghaoui, E. Feron, and V. Balakrishnan. Linear Matrix Inequalities in System and Control Theory, volume 15 of Studies in Applied Mathematics. SIAM, Philadelphia, PA, June 1994.

4. Jérôme Feret. Static analysis of digital filters. In European Symposium on Programming (ESOP'04), number 2986 in LNCS. Springer-Verlag, 2004.

5. E. Feron. From control systems to control software. Control Systems, IEEE, 30(6):50 -71, dec. 2010.

6. Eric Goubault and Sylvie Putot. A zonotopic framework for functional abstractions. CoRR, abs/0910.1763, 2009.

7. Heber Herencia-Zapana, Romain Jobredeaux, Sam Owre, Pierre-Loïc Garoche, Eric Feron, Gilberto Perez, and Pablo Ascariz. Pvs linear algebra libraries for verification of control software algorithms in c/acsl. In NASA Formal Methods, pages 147-161, 2012.

8. C. A. R. Hoare. An axiomatic basis for computer programming. Commun. ACM, 12:576-580, October 1969.

9. Nassima Izerrouken, Marc Pantel, Xavier Thirioux, and Olivier Ssi Yan Kai. Integrated formal approach for qualified critical embedded code generator. In Formal Methods for Industrial Critical Systems, volume 5825 of Lecture Notes in Computer Science, pages 199-201. Springer Berlin Heidelberg, 2009.

10. A. Megretski and A. Rantzer. System analysis via integral quadratic constraints. Automatic Control, IEEE Transactions on, 42(6):819-830, 1997.

11. Sam Owre and N. Shankar. Theory interpretation in pvs. Technical report, SRI International, 2001.

12. Pierre Roux, Romain Jobredeaux, Pierre-Loïc Garoche, and Eric Feron. A generic ellipsoid abstract domain for linear time invariant systems. In $H S C C$, pages 105114, 2012.

13. V Ao Yakubovich. S-procedure in nonlinear control theory. Vestnik Leningrad University, 1:62-77, 1971.

14. Vladimir A Yakubovich. The solution of certain matrix inequalities in automatic control theory. In Soviet Math. Dokl, volume 3, pages 620-623, 1962. 